\title{
GESTÃO DA MARCA EM IES: UM ESTUDO BIBLIOMÉTRICO
}

\author{
Ramon Alves de Oliveira* \\ Danilo de Melo Costa*
}

Resumo: Este artigo pretende realizar uma análise bibliométrica do estado atual da pesquisa de "marca" e "gestão da marca", buscando identificar autores e países mais produtivos ao longo do tempo, análise de publicações, cooperação entre autores, co-ocorrências de palavras-chave para visualizar a estrutura conceitual do campo do conhecimento. Utilizou-se a base de dados Scopus Elsevier, contemplada no Portal Capes. Os seis grandes grupos de investigação contemplaram as palavras: "Brand"; "Brand $\mathrm{Ma}$ nagement"; "Brand Hatred"; "Brand Loyalty"; "Brand and Educational Institution"; "Brand Management and Educational Institution". A proposta de pesquisa foi: TITLE-ABS-KEY a fim de levar em consideração diferentes pesquisas que contemplaram o construto. Percebe-se que as temáticas aqui abordadas contribuem para a pesquisa acadêmica em quantidade, mediante número de publicações e relevância, mediante os trabalhos citados. Não obstante, a análise bibliométrica também permitiu concluir que há uma lacuna ao observar a busca pelas palavras-chave Brand hatred; Hate the Brand; e Education Institution, as quais não constaram publicações na base de dados Scopus. Diante dessa lacuna, sugere-se que sejam realizados estudos voltados para o ódio à marca e o ódio à marca em instituições de ensino e gestão da marca como fator de estratégia de retenção e promoção da lealdade e fidelização de alunos.

Palavras-chave: Marca; Instituição de Ensino; Branding.

\footnotetext{
* Mestre em Turismo e Meio Ambiente - Centro Universitário UNA (Brasil). Mestre em Administração - Fundação Pedro Leopoldo - FPL (Brasil). Doutorando em Administração - Universidade FUMEC-BH (Brasil). Professor UNIMONTES e UNIFIPMoc.

E-mail: ramonalves@yahoo.com.br

${ }^{* *}$ Doutor em administração pela Universidade Federal de Minas Gerais - UFMG (Brasil) com período sanduíche na York University (Canadá). Professor do Programa de Doutorado e Mestrado em Administração (PDMA) da Universidade FUMEC. danilomct@gmail.com
} 


\begin{abstract}
This article intends to carry out a bibliometric analysis of the current state of research on "brand" and "brand management", seeking to identify authors and more productive countries over time, analysis of publications, cooperation between authors, co-occurrences of keywords to visualize the conceptual structure of the field of knowledge. The Scopus Elsevier database, used in the Capes Portal, was used. The six major research groups contemplated the words: "Brand"; "Brand Management"; "Brand Hatred"; "Brand Loyalty"; "Brand and Educational Institution"; "Brand Management and Educational Institution". The research proposal was: TITLE-ABS-KEY in order to take into account different research that contemplated the construct. It is noticed that the themes addressed here contribute to academic research in quantity, through number of publications and relevance, through the works cited. Nevertheless, bibliometric analysis also allowed us to conclude that there is a gap when observing the search for the keywords Brand hatred; Hate the Brand; and Education Institution, which did not include publications in the Scopus database. In view of this gap, it is suggested that studies on brand hatred and brand hatred be carried out in educational institutions and brand management as a factor for retaining and promoting student loyalty and loyalty.
\end{abstract}

Keywords: Brand; Educational Institution; Branding.

Resumen: Este artículo pretende realizar un análisis bibliométrico del estado actual de la investigación sobre "marca" y "gestión de marca", buscando identificar autores y países más productivos en el tiempo, análisis de publicaciones, cooperación entre autores, co-ocurrencia de palabras clave para visualizar la estructura conceptual del campo del conocimiento. Se utilizó la base de datos Scopus Elsevier, utilizada en el Portal de Capes. Los seis grandes grupos de investigación contemplaron las palabras: "Marca"; "Gestión de la marca"; "Odio a las marcas"; "Lealtad a la marca"; "Marca e Institución Educativa"; "Gestión de Marca e Institución Educativa" La propuesta de investigación fue: TÍTULO-ABS-CLAVE para tomar en cuenta las diferentes investigaciones que contemplaron el constructo. Se advierte que los temas aquí abordados contribuyen a la investigación académica en cantidad, por número de publicaciones y relevancia, a través de los trabajos citados. Sin embargo, el análisis bibliométrico también nos permitió concluir que existe un vacío al observar la búsqueda de las palabras clave Odio a la marca; Odio la marca; e Institución Educativa, que no incluyó publicaciones en la base de datos Scopus. Ante esta brecha, se sugiere que se realicen estudios sobre el odio a la marca y el odio a la marca en las instituciones educativas y la gestión de marca como factor de retención y promoción de la lealtad y fidelización de los estudiantes.

Palabras llave: Marca; Institucion de enseñanza; Marca. 


\section{Introdução}

Os estudos sobre gestão da marca contemplam a pesquisa nas Ciências Sociais Aplicadas desde 1981, sendo a primeira pesquisa identificada o modelo lógico de comportamento do consumidor proposto por Zufryden (1981), o qual seu estudo descreve e avalia empiricamente uma abordagem para modelar o comportamento de compra que integra uma estrutura de modelo de escolha de marca multivariada baseada em Logit-Markov com componentes estocásticos.

Após a pesquisa de Zufryden (1981), mediante um estudo bibliométrico realizado na base de dados Scopus, identificou-se um total de 2441 (dois mil quatrocentos e quarenta e um) estudos que contemplam a temática de gestão da marca.

O número considerável de publicações demonstra que este tema tem trazido discussões para a academia e sua importância no contexto da pesquisa nas Ciências Sociais Aplicadas. Entre 1981 e 2021, o ano que obteve um maior número de publicações sobre a gestão da marca foi o ano de 2011, contemplando 219 estudos.

A proposta deste artigo permeia a gestão da marca em Instituições de Ensino Superior (IES), com foco na mediação do ódio à marca na decisão de abandono ou permanência. Diante disso, o estudo do branding em instituições de ensino também é importante para ancorar a pesquisa proposta.

Diante do exposto, este artigo pretende realizar um estudo contemplando uma análise bibliométrica do estado atual da pesquisa de "marca" e "gestão da marca", mapeando o cenário de pesquisa para identificar autores e países mais produtivos ao longo do tempo, análise de publicações, cooperação entre autores e co-ocorrências de palavras-chave para visualizar a estrutura conceitual do campo do conhecimento.

Usando a coleção Scopus, este estudo analisa 2000 publicações que estudam a marca e a gestão da marca, considerando também instituições de ensino superior. 
Este artigo contempla a introdução com a contextualização da temática, a metodologia, apresentando os passos para a realização da pesquisa e os resultados contemplando uma análise das publicações buscadas na base de dados utilizada.

\section{Metodologia}

Para a análise bibliométrica, utilizou-se a base de dados Scopus Elsevier, contemplada no Portal Capes.

Os seis grandes grupos de investigação contemplaram as palavras: "Brand"; "Brand Management"; "Brand Hatred"; "Brand Loyalty"; "Brand and Educational Institution"; "Brand Management and Educational Institution".

A proposta de pesquisa foi: TITLE-ABS-KEY a fim de levar em consideração diferentes pesquisas que contemplaram o construto. A pesquisa foi enquadrada nos documentos disponiveis na base de dados, que eram 2.000 documentos e o período de tempo analisado estendeu-se de 2000 até 2021 , tendo em conta que a procura pelas informações foram realizadas no período de 06 de novembro a 07 de Dezembro de 2020.

O levantamento de dados foi realizado com os softwares VosViewer $\square$ e Microsoft Excel®.

\section{Resultados e Discussão}

Essa pesquisa fornece uma visão geral que pode ser um guia importante para pesquisadores da área de marketing que realizam pesquisa sobre o Branding. As análises seguintes compreendem o resultado de uma busca pelas principais palavras-chave relacionadas à temática da pesquisa.

A Tabela 1 mostra o número de artigos publicados no período de 2000 a 2021 que contemplam as palavras-chave pesquisadas no título, no resumo ou nas palavras-chave. 
Tabela 1 - Número de artigos publicados com as palavras-chave pesquisadas

\begin{tabular}{|c|c|c|c|c|c|c|}
\hline \multirow[b]{2}{*}{ Ano } & \multicolumn{6}{|c|}{ Palavras-chave buscadas } \\
\hline & Brand & $\begin{array}{l}\text { Brand } \\
\text { Management }\end{array}$ & $\begin{array}{l}\text { Brand } \\
\text { Hatred }\end{array}$ & $\begin{array}{l}\text { Brand } \\
\text { Loyalty }\end{array}$ & $\begin{array}{l}\text { Brand } \\
\text { and } \\
\text { IES }\end{array}$ & $\begin{array}{l}\text { Brand } \\
\text { Management } \\
\text { and IES }\end{array}$ \\
\hline 2021 & 102 & 9 & 0 & 6 & 13 & 0 \\
\hline 2020 & 5642 & 732 & 3 & 431 & 18 & 11 \\
\hline 2019 & 5380 & 727 & 1 & 448 & 9 & 14 \\
\hline 2018 & 4526 & 602 & 0 & 339 & 12 & 9 \\
\hline 2017 & 3962 & 538 & 0 & 266 & 9 & 10 \\
\hline 2016 & 3791 & 501 & 0 & 275 & 6 & 8 \\
\hline 2015 & 3646 & 446 & 1 & 225 & 16 & 4 \\
\hline 2014 & 3413 & 456 & 0 & 223 & 4 & 6 \\
\hline 2013 & 3151 & 455 & 0 & 188 & 2 & 3 \\
\hline 2012 & 2823 & 387 & 0 & 185 & 6 & 1 \\
\hline 2011 & 2738 & 404 & 0 & 156 & 2 & 4 \\
\hline 2010 & 2545 & 356 & 1 & 143 & 1 & 3 \\
\hline 2009 & 2382 & 352 & 0 & 124 & 2 & 2 \\
\hline 2008 & 2283 & 296 & 0 & 95 & 1 & 0 \\
\hline 2007 & 2292 & 296 & 1 & 89 & 2 & 2 \\
\hline 2006 & 1815 & 254 & 0 & 60 & 1 & 2 \\
\hline 2005 & 1452 & 200 & 0 & 58 & 1 & 1 \\
\hline 2004 & 1338 & 135 & 1 & 46 & 1 & 1 \\
\hline 2003 & 1068 & 116 & 0 & 39 & 0 & 2 \\
\hline 2002 & 1115 & 116 & 0 & 28 & 0 & 0 \\
\hline 2001 & 940 & 113 & 1 & 38 & 0 & 0 \\
\hline 2000 & 1092 & 99 & 0 & 32 & 0 & 0 \\
\hline TOTAL & 57496 & 7590 & 9 & 2609 & 106 & 83 \\
\hline
\end{tabular}

Fonte: Adaptada de SCOPUS, 2020.

A partir dos dados expostos na Tabela 1, observa-se que a palavra-chave "Brand" contempla o maior número de artigos publicados no período de 2000 a 2021, sendo 57496 (cinquenta e sete mil quatrocentos e noventa e seis) no total, com destaque ao ano de 2020, com 5642 artigos publicados.

A Tabela 1 também permite inferir que a partir do ano de 2003, o número de artigos publicados têm aumentado ano a ano, o que demonstra a importância do tema. 
As demais buscas, "Brand Management", "Brand Hatred", "Brand Loyalty", "Brand and IES" e "Brand Management and IES", obtiveram 7590, 9, 2609, 106 e 83 artigos publicados no período analisado, respectivamente.

Para uma maior compreensão acerca do construto que compreende a temática desta pesquisa, foram feitas análises sobre cada palavra-chave.

\subsection{Palavra-chave "Brand"}

A Tabela 2 mostra a lista dos dez (10) autores mais produtivos cujos artigos publicados contemplam a palavra "Brand", no período analisado.

Tabela 2 - 10 autores mais produtivos

\begin{tabular}{l|r}
\hline Autor & $\mathbf{N}^{\circ}$ de Publicações \\
\hline Phau, I. & 56 \\
Romaniuk, J. & 52 \\
Merrilees, B. & 51 \\
Hammond, D. & 47 \\
Fong, G.T. & 46 \\
Lee, Y.K.. & 46 \\
Melewar, T.C.. & 46 \\
Anon. & 43 \\
O'Connor, R.J. & 43 \\
Sung, Y.. & 41 \\
\hline
\end{tabular}

Fonte: Adaptada de SCOPUS, 2020.

A classificação exposta na Tabela 2 é baseada no número total de publicações do autor e não na ordem de autoria. O autor Phau, I. lidera com 56 publicações entre os anos de 2000 e 2021, seguido por Romaniuk, J. $(n=52)$ e Merriles, B. $(n=51)$. 


\section{Figura 1 - Mapa de análise de citação}

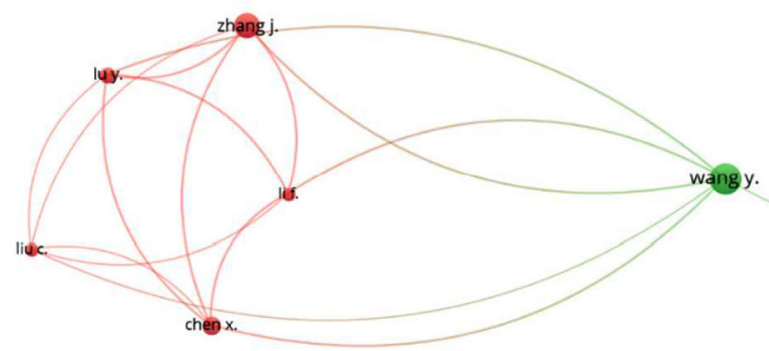

A vosviewer

Fonte: Adaptada de SCOPUS, 2020.

A Figura 1 demonstra os autores mais citados que contemplam em seus artigos a palavra-chave "Brand". De forma diferente, ao contemplar o número de citações, conforme exposto na Figura 1, a liderança em citações fica com o autor Wang, Y., o qual dispõe de maior concentração no mapa.

Além do mapa de citação também foi elaborada uma tabela (Tabela 3) contemplando os dez (10) autores mais citados.

Tabela 3 - Autores mais citados

\begin{tabular}{lrrr}
\hline Autor & $\begin{array}{l}\text { Artigos } \\
\text { publicados }\end{array}$ & $\mathbf{N}^{\circ}$ de citações \\
\hline Wang, y & 27 & 59 \\
Li, h. & 10 & 30 \\
Li, j. & 17 & 26 \\
Chen, h & 8 & 22 \\
Kim, y. & 9 & 22 \\
Wang, x. & 9 & 21 \\
Li, f. & 5 & 20 \\
Liu y. & 16 & 19 \\
Li, y & 21 & 16 \\
Kim, j. & 11 & 14 \\
\hline
\end{tabular}

Fonte: Adaptada de SCOPUS, 2020.

De acordo com a Tabela 3, o autor Wang, Y. é o mais citado entre os resultados a partir da busca da palavra "Brand". No entanto, este autor 
não contempla a lista dos mais produtivos, pois publicou 27 artigos com as características (palavra-chave e período) analisadas na busca.

Outro ponto importante a ser analisado é o território onde o documento foi publicado.

Tabela 4 - Publicação por território

\begin{tabular}{l|r}
\hline Pais & $\mathbf{N}^{\circ}$ de Publicações \\
\hline United States & 14047 \\
China & 4901 \\
United Kingdom & 4493 \\
India & 3154 \\
Australia & 2666 \\
Spain & 2145 \\
Germany & 2001 \\
Canada & 1842 \\
Brazil & 1683 \\
South Korea & 1621 \\
\hline
\end{tabular}

Fonte: Adaptada de SCOPUS, 2020.

Foram identificados 159 territórios com publicações cujas características contemplam a busca na base de dados. Entre os países identificados, a Tabela 4 mostra os dez (10) mais produtivos, com destaque aos Estados Unidos, China e Reino Unido. O Brasil se encontra na nona $\left(9^{\mathrm{a}}\right)$ posição com 1683 artigos publicados no período analisado. 


\section{Figura 2 - Mapa de co-ocorrência de palavras}

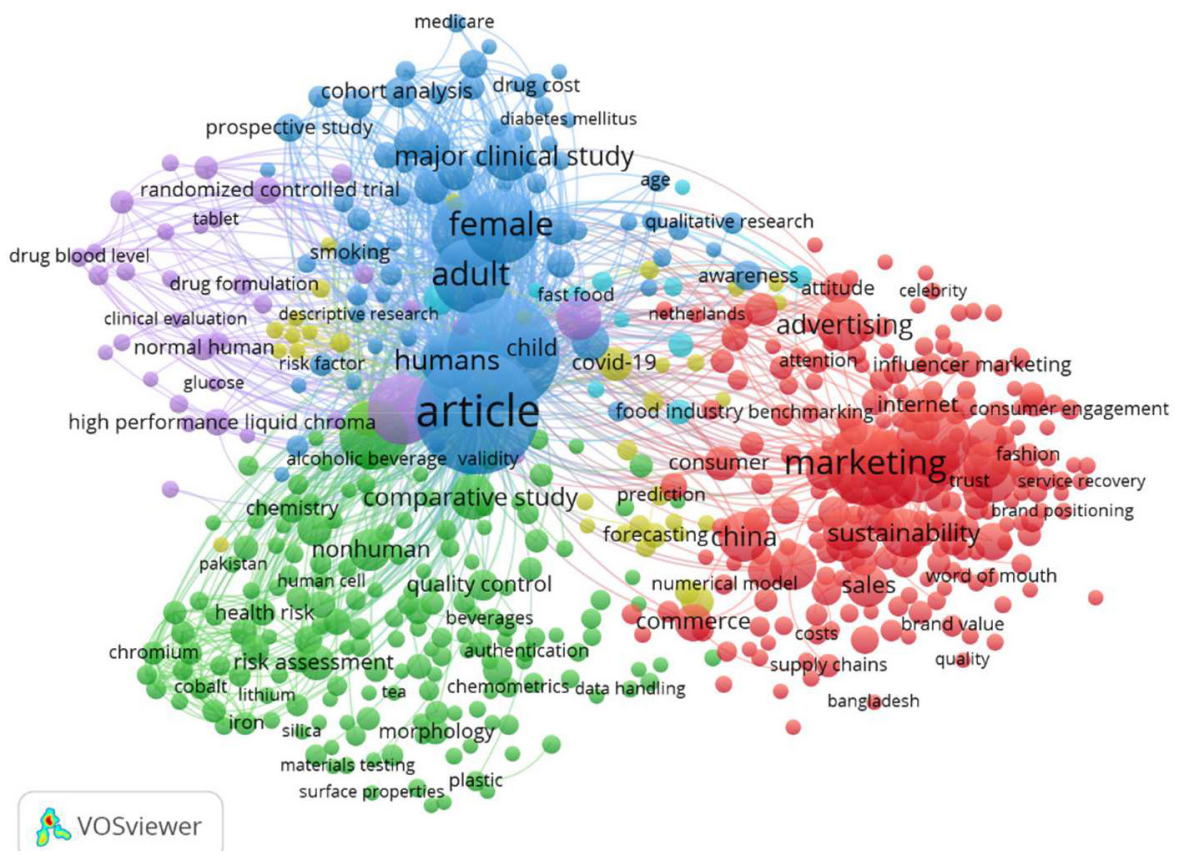

Fonte: Adaptada de SCOPUS, 2020.

A Figura 2 mostra a densidade das palavras que co-ocorrem nos artigos buscados. Observa-se que as palavras Marketing, Sustainability, Humans e Article são as que mais ocorrem.

Este resultado demonstra que os autores que pesquisam sobre Brand também discutem sobre estes outros conceitos como o Brand Management, sendo esta uma palavra-chave também pesquisada neste estudo bibliométrico.

\subsection{Palavra-chave Brand Management}

Ao contemplar a palavra Brand Management, identificou-se publicações desde 1959 até o ano 2021. No entanto, o período escolhido para a análise permeia o período de 2000 a 2021 , contemplando 7590 artigos.

A primeira análise, exposta na Tabela 5, consiste nos autores mais produtivos. 
Tabela 5 - Autores mais produtivos

\begin{tabular}{l|r}
\hline Autor & No de publicações \\
\hline King, C. & 26 \\
Merrilees, B., & 16 \\
Gupta, S. & 15 \\
Abratt, R. & 14 \\
Melewar, T.C. & 14 \\
Foroudi, P. & 12 \\
Grace, D. & 12 \\
Rubio, N. & 12 \\
Balmer, J.M.T. & 11 \\
Miller, D. & 11 \\
\hline
\end{tabular}

Fonte: Adaptada de SCOPUS, 2020.

Entre os 10 mais produtivos, King, C lidera com 26 artigos publicados, enquanto Miller, D. fica em décimo com 11 publicações.

A Tabela 6 apresenta os dez (10) autores mais citados cujos artigos contemplam a palavra Brand Management.

Tabela 6 - Autores mais citados

\begin{tabular}{l|r}
\hline Autor & N $^{\circ}$ de citações \\
\hline Liu y & 69 \\
\hline Wang y. & 65 \\
\hline Jin s. v. & 61 \\
\hline Gupta s. & 59 \\
\hline Chen y. & 58 \\
\hline Foroudi & 57 \\
p. & 44 \\
\hline Chen j. & 44 \\
\hline He y. & 42 \\
\hline Xiong 1. & 41 \\
\hline King c. & \\
\hline
\end{tabular}

Fonte: Adaptada de SCOPUS, 2020.

De forma díspar ao número de publicações (Tabela 5), ao tratar o número de citações, o autor King, C. está em $10^{\circ}$ lugar com 41 citações, enquanto o líder é o autor Liu, Y. com 69 citações. 


\section{Figura 3 - Mapa de análise de citação}

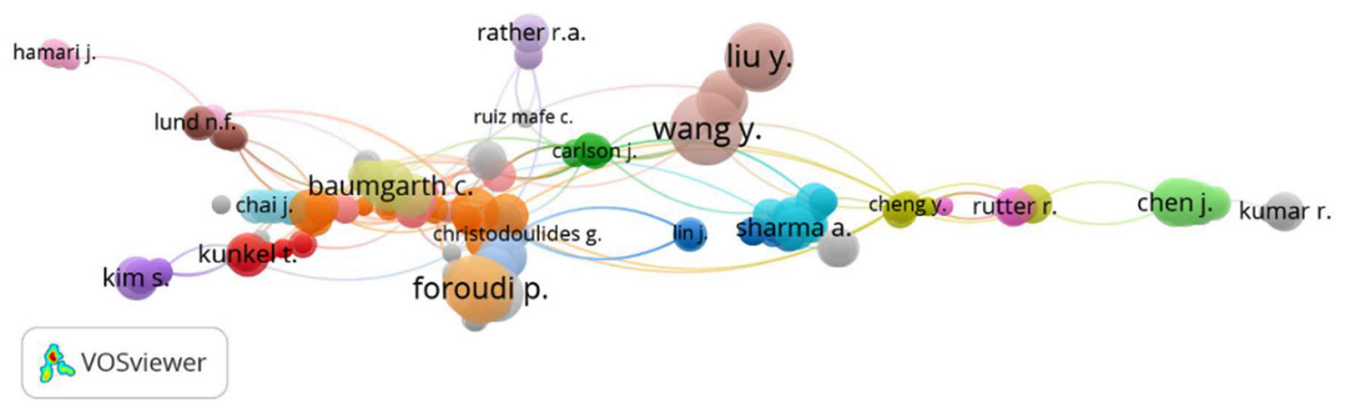

Fonte: Adaptada de SCOPUS, 2020.

A Figura 3 permite a análise acerca dos autores mais citados, sendo estes os que apresentam maior densidade no mapa, os quais são Wang, Y.; Liu, Y.; Foroudi, p. e outros. Observou-se que os autores Wang, Y. e Liu, Y. também foram citados na busca da palavra-chave "Brand".

Além dos autores mais citados, a co-ocorrência de palavras é importante em uma análise bibliométrica.

\section{Figura 4 - Mapa de co-ocorrência de palavras}

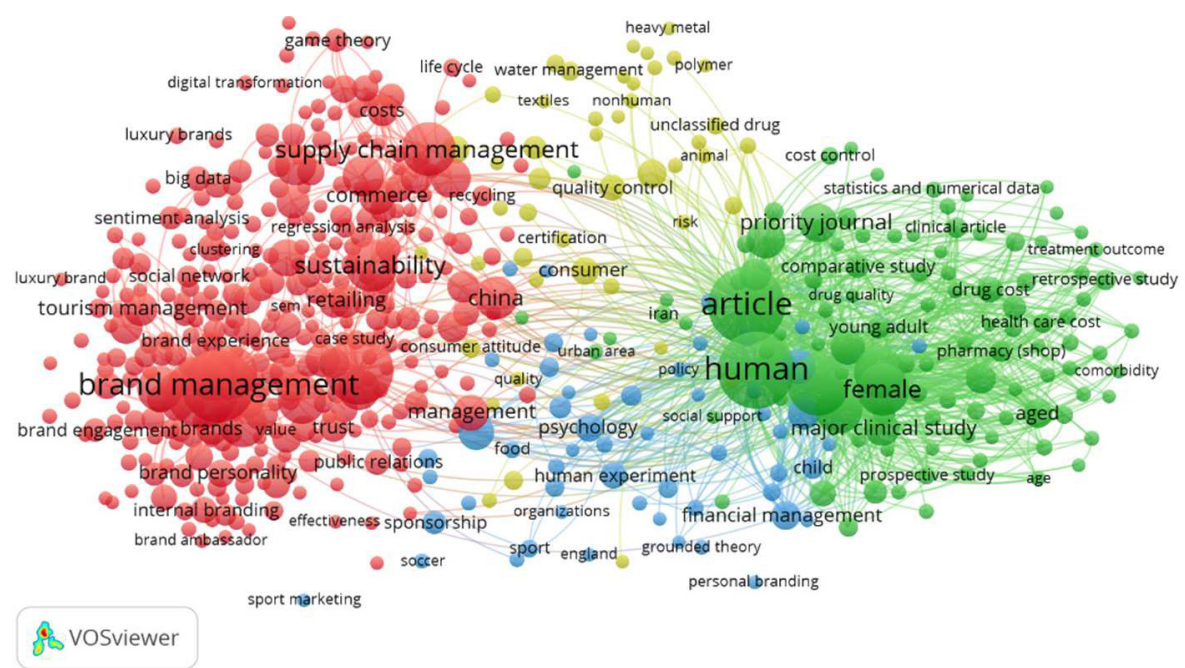

Fonte: Adaptada de SCOPUS, 2020.

A Figura 4 contempla a co-ocorrência de palavras nos artigos buscados. Observa-se que as palavras Brand management e supply chain management apresentam uma boa densidade e também as palavras Human, 
article e female, sendo as últimas também citadas nos artigos buscados pela palavra-chave Brand.

\subsection{Palavra-chave: Brand hatred}

A partir da palavra-chave Brand hatred foram obtidos resultados de 16 documentos publicados desde o ano de 1994. Ao limitar o período de busca de 2000 a 2020, foram obtidos 9 artigos.

\section{Tabela 7 - Autores e número de publicações}

\begin{tabular}{l|r}
\hline Autor & $\mathbf{N}^{\circ}$ de artigos publicados \\
\hline Brandão, A. & 1 \\
Dame-Griff, E.C. & 1 \\
Gräfe, T. & 1 \\
Hau, C.S. & 1 \\
Hibberd, J. & 1 \\
Kucuk, S.U. & 1 \\
Morando, M. & 1 \\
Osseiran-Houbballah, & 1 \\
M. & 1 \\
Platania, S. & \\
\hline
\end{tabular}

Fonte: Adaptada de SCOPUS, 2020.

Conforme exposto na Tabela 7 , todos os autores publicaram a mesma quantidade de artigos no período analisado, portanto não há a possibilidade de elencar o mais produtivo por número de publicações.

Diante disso, foi realizada a análise de coautoria, exposta na Figura 5. 
Figura 5 - Análise de coautoria

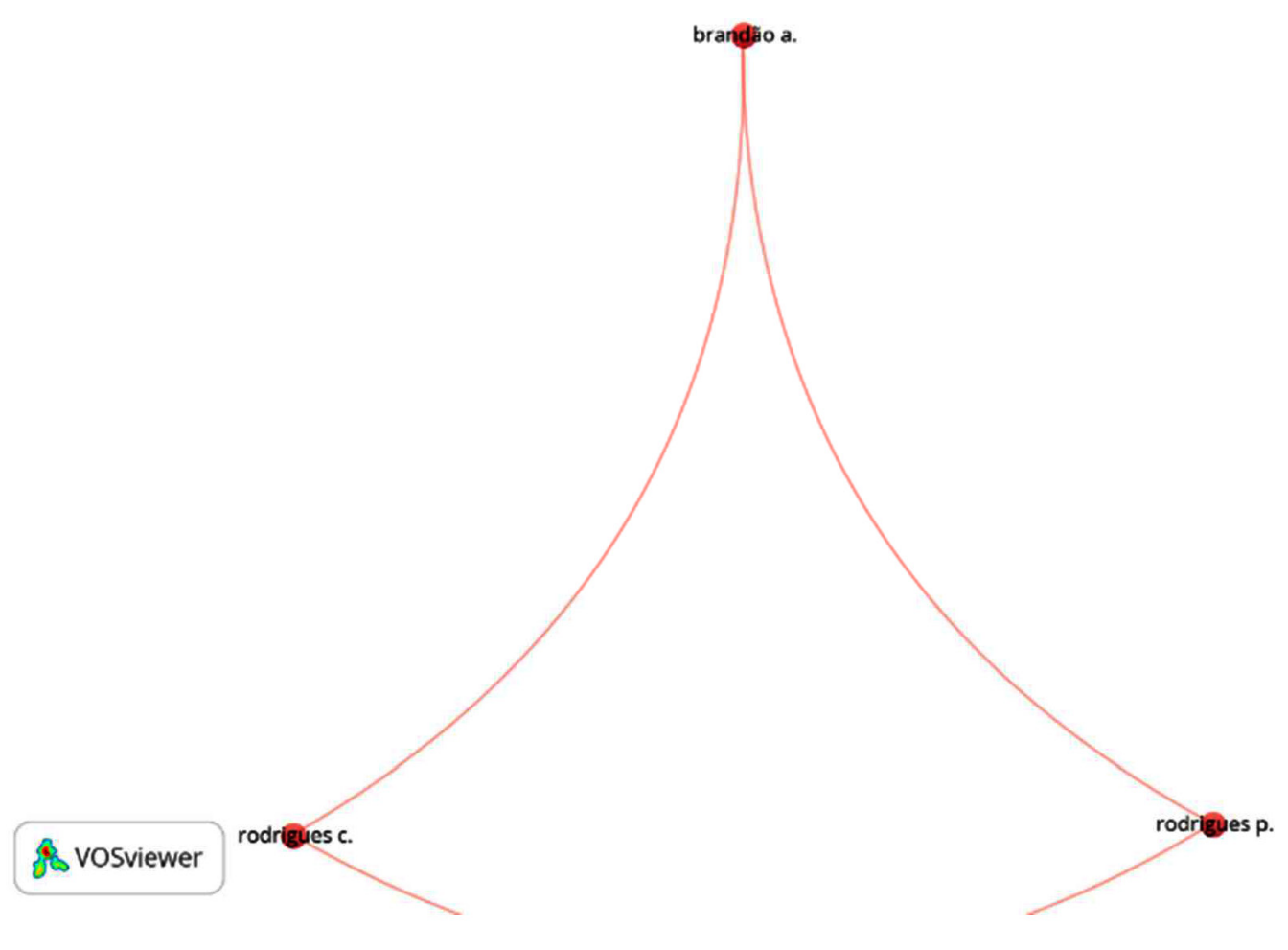

Fonte: Adaptada de Vosviewer/SCOPUS, 2020.

A partir da análise de coautoria (Figura 5), observa-se três principais autores que realizaram pesquisas juntos sobre a temática do ódio à marca (Brand Hatred), quais sejam: Brandão, A.; Rodrigues, C; e Rodrigues, P.

Quanto à citação, os autores supracitados não apresentam destaque (Tabela 8). 
Tabela 8 - Análise de citação

\begin{tabular}{lccc}
\hline Autor & Documentos & $\begin{array}{c}\mathbf{N}^{\circ} \mathbf{d e} \\
\text { citações }\end{array}$ \\
\hline Tan e.k.b. & 1 & 16 \\
Kucuk s.u. & 1 & 11 \\
Hau c.s. & 1 & 3 \\
Grafe t. & 1 & 1 \\
Brandão a. & 1 & 0 \\
Rodrigues c. & 1 & 0 \\
Rodrigues p. & 1 & 0 \\
Drame-griff e.c. & 1 & 0 \\
Hibberd j. & 1 & 0 \\
Itani o.s. & 1 & 0 \\
Morando m. & 1 & 0 \\
Osseiran-Houbballah & & 0 \\
m. & 1 & 0 \\
Platania s. & 1 & 0 \\
Santisi g. & 1 & \\
\hline
\end{tabular}

Fonte: Adaptada de Vosviewer/SCOPUS, 2020.

Conforme exposto na Tabela 8, Tan e.k.b. e Kucuk s.u. são os autores que apresentam maior número de citações em outros trabalhos, sendo 16 e 11 respectivamente.

\section{Figura 6 - Mapa de co-ocorrência de palavras}

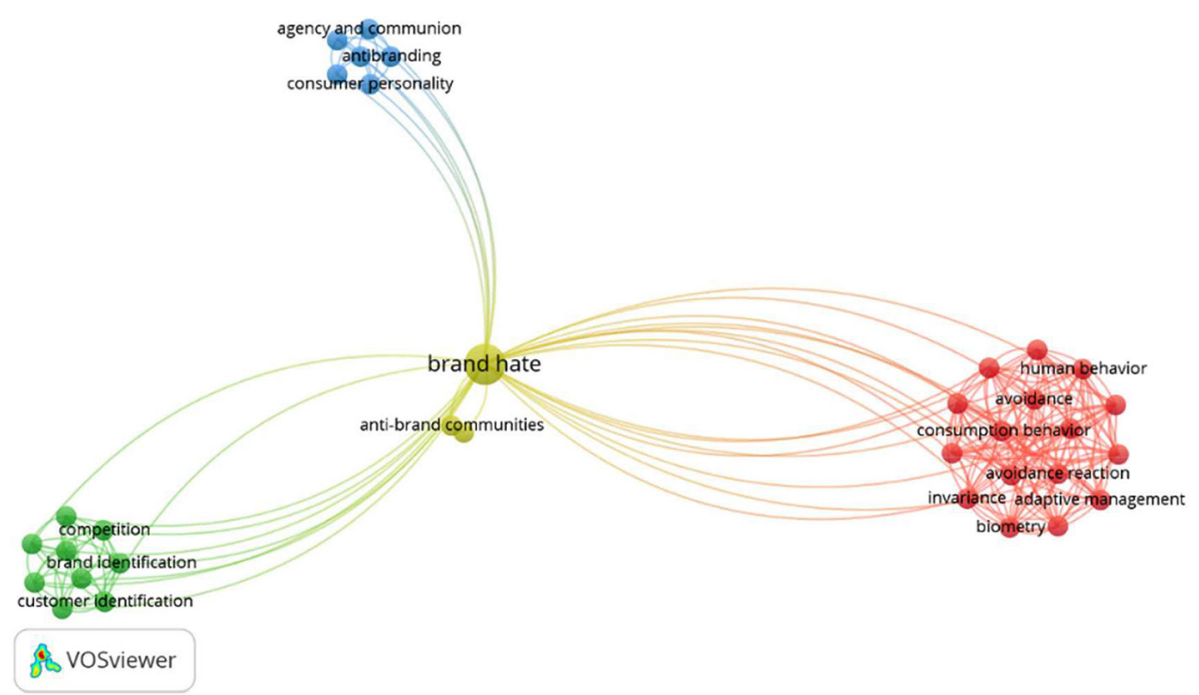

Fonte: Adaptada de Vosviewer/SCOPUS, 2020. 
Quanto à co-ocorrencia de palavras-chave, as palavras brand hate e anti-brand communities estão no centro do mapa, o que representa a sua correlação com os demais grupos de palavras.

Tabela 9 - Países e número de publicações

\begin{tabular}{l|r}
\hline País & $\mathbf{N}^{\mathbf{0}}$ de artigos publicados \\
\hline United States & 2 \\
France & 1 \\
Italy & 1 \\
Japan & 1 \\
Portugal & 1 \\
Singapore & 1 \\
Sweden & 1 \\
United & 1 \\
Kingdom & 1 \\
Undefined & \\
\hline \multicolumn{2}{l}{ Fonte: Adaptada de Vosviewer/SCOPUS, 2020. }
\end{tabular}

A Tabela 9 apresenta o número de publicações em relação aos países que contemplam pesquisas voltadas pata o Brand hatred, tendo como destaque mais uma vez os Estados Unidos.

\subsection{Palavra-chave: Brand Loyalty}

O estudo sobre marca e gestão da marca contempla também a lealdade à marca (Brand Loyalty). Diante disso, a palavra-chave Brand loyalty foi inserida na busca na base de dados.

Foram identificados 4613 documentos publicados entre 1944 e 2021, sendo 3494 artigos publicados no período de 2000 a 2021. 
Tabela 10 - Publicações por ano

\begin{tabular}{l|r}
\hline Ano & $\mathbf{N}^{\circ}$ de artigos publicados \\
\hline 2021 & 6 \\
2020 & 431 \\
2019 & 448 \\
2018 & 339 \\
2017 & 266 \\
2016 & 275 \\
2015 & 225 \\
2014 & 223 \\
2013 & 188 \\
2012 & 185 \\
2011 & 156 \\
2010 & 143 \\
2009 & 124 \\
2008 & 95 \\
2007 & 89 \\
2006 & 60 \\
2005 & 58 \\
2004 & 46 \\
2003 & 39 \\
2002 & 28 \\
2001 & 38 \\
2000 & 32 \\
\hline
\end{tabular}

Fonte: Adaptada de SCOPUS, 2020.

Conforme os dados expostos na Tabela 10, o ano com maior número de publicações que contemple o tema Brand Loyalty foi 2019 seguido de 2020.

A Tabela 11 mostra os autores com maior produção no período analisado. 
Tabela 11 - Autores mais produtivos

\begin{tabular}{l|r}
\hline Autor & $\mathbf{N}^{\circ}$ de artigos publicados \\
\hline Dawes, J. & 16 \\
Han, H. & 15 \\
Gil-Saura, I. & 13 \\
Rahman, Z. & 13 \\
Back, K.J. & 12 \\
Nguyen, B. & 11 \\
Šerić, M. & 11 \\
Ahn, J. & 10 \\
Flavián, C. & 10 \\
Khan, I. & 10 \\
Kim, Y.K. & 10 \\
\hline
\end{tabular}

Fonte: Adaptada de SCOPUS, 2020.

Conforme exposto na Tabela 11, o autor Dawes, J. obteve o maior número de artigos publicados sobre Brand Loyalty publicados entre 2000 e 2021.

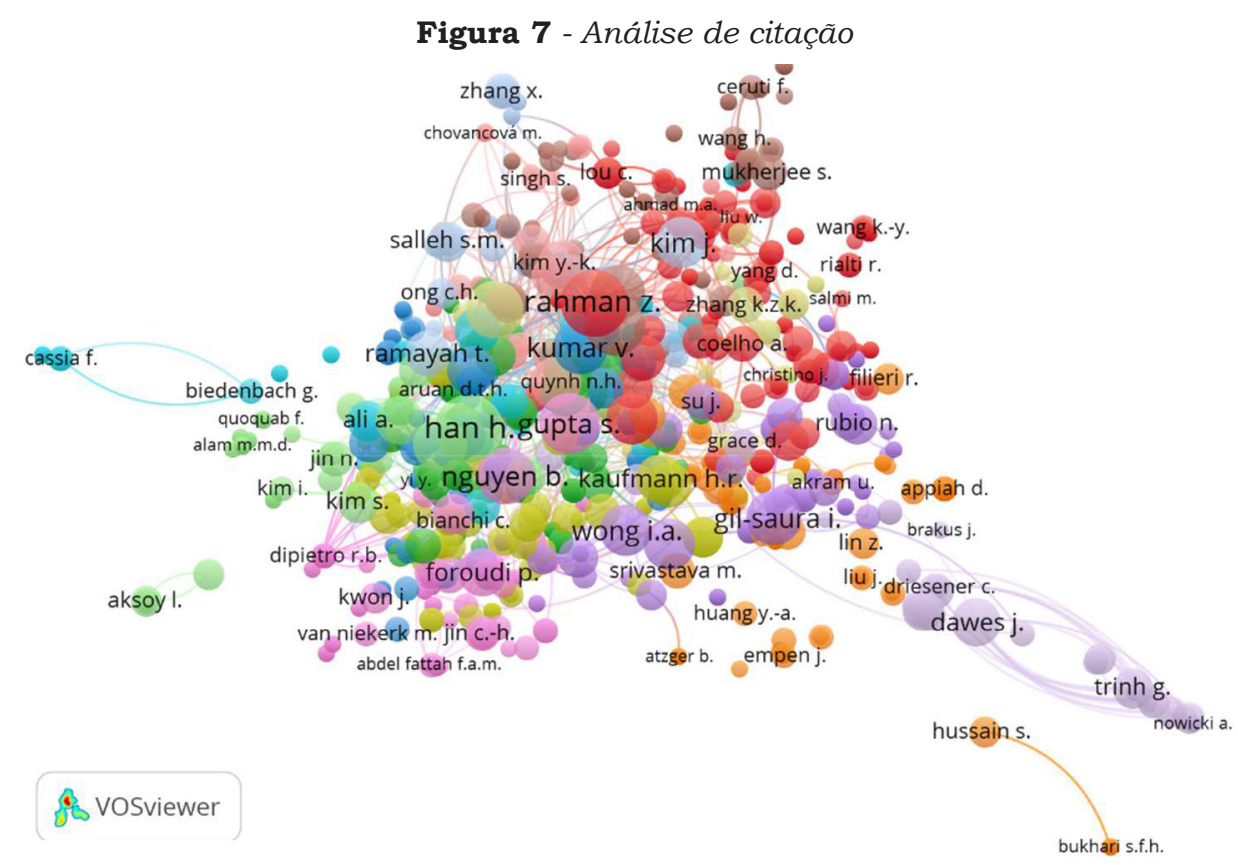

Fonte: Adaptada de Vosviewer/SCOPUS, 2020. 
Tabela 12 - Análise de citações

\begin{tabular}{lrrr}
\hline Autor & Documentos & \multicolumn{1}{c}{$\begin{array}{c}\mathbf{N}^{\mathbf{d}} \mathbf{d e} \\
\text { citações }\end{array}$} \\
\hline BILGIHAN A. & 5 & 396 \\
RAHMAN Z. & 13 & 383 \\
NYADZAYO & & \\
M.W. & 5 & 277 \\
WANG Y & 8 & 273 \\
KING C & 4 & 263 \\
SO K.K.F. & 4 & 255 \\
SPARKS B.A. & 2 & 255 \\
GUPTA S. & 11 & 244 \\
LECKIE C. & 7 & 235 \\
MANTHIOU A. & 2 & 207 \\
\hline
\end{tabular}

Fonte: Adaptada de Vosviewer/SCOPUS, 2020.

A Figura 12 e a Tabela 12 contemplam a análise de citações, por meio dos quais é possivel observar que Bilgihan, A. é o autor mais citado, com 396 citações e cinco (5) documentos publicados no período analisado.

Os 10 países com maior número de publicações que compreendem a Brand Loyalty estão dispostos na Tabela 13 .

Tabela 13- Países com maior número de publicações

\begin{tabular}{l|c}
\hline Pais & $\mathbf{N}^{\mathbf{0}}$ de artigos publicados \\
\hline United States & 833 \\
India & 350 \\
United Kingdom & 298 \\
Australia & 256 \\
South Korea & 198 \\
Spain & 188 \\
China & 179 \\
Taiwan & 160 \\
Malaysia & 156 \\
Iran & 109 \\
\hline
\end{tabular}

Fonte: Adaptada de Vosviewer/SCOPUS, 2020. 


\section{Figura 8 - Mapa de co-ocorrência de palavras}

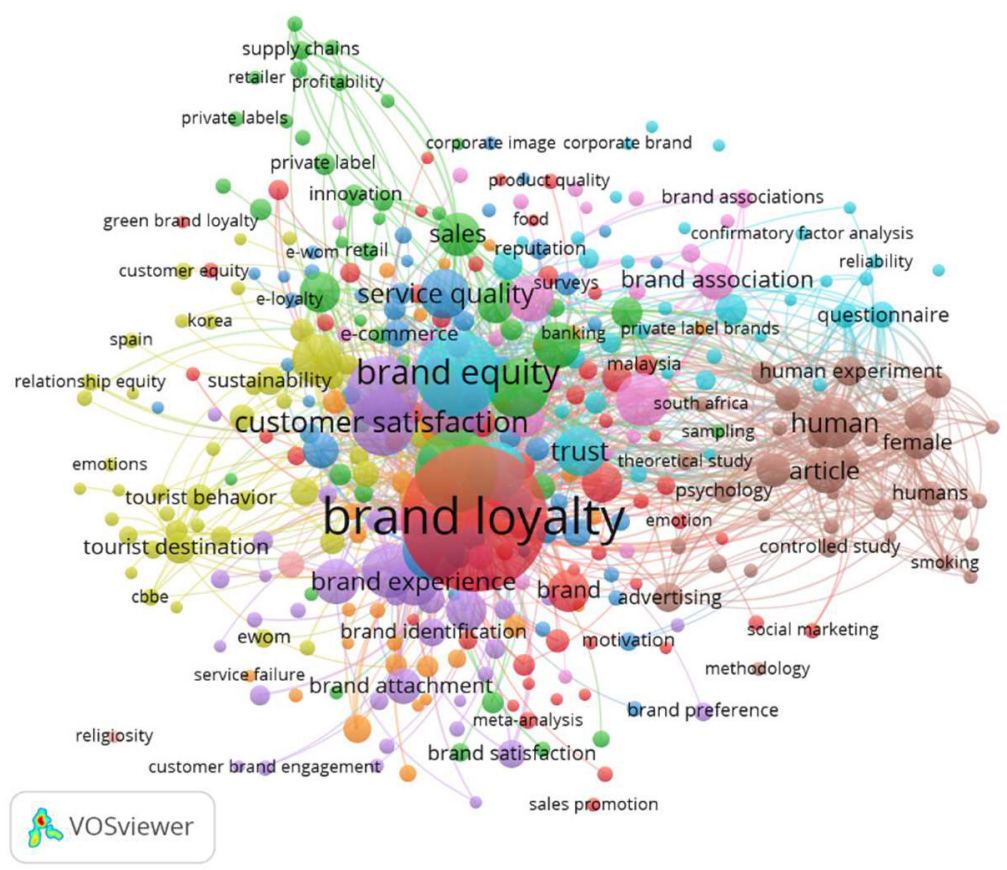

Fonte: Adaptada de Vosviewer/SCOPUS, 2020.

Quanto à co-ocorrência de palavras nos trabalhos buscados com a palavra pesquisada (Brand loyalty), as palavras Brand equity e customer satisfaction foram as mais citadas nos trabalhos apresentados no levantamento.

\subsection{Palavra-chave Brand and Education Institution}

Mediante à proposta de pesquisa deste projeto, a palavra-chave "Brand and Education Institution" se faz importante devido ao fato do objeto de pesquisa consistir em Instituições de Ensino Superior.

Por meio da busca da palavra-chave "Brand and Education Institution" foram identificados 172 documentos no período de 1940 a 2020 . Ao filtrar o período de 2000 a 2021, foram identificados 106 artigos no período de 2003 a 2020.

A Tabela 14 contempla o número de publicações por ano analisado. 
Tabela 14 - Número de publicações por ano

\begin{tabular}{lr}
\hline Ano & $\mathbf{N}^{\circ}$ de artigos publicados \\
\hline 2020 & 13 \\
2019 & 18 \\
2018 & 9 \\
2017 & 12 \\
2016 & 9 \\
2015 & 6 \\
2014 & 16 \\
2013 & 4 \\
2012 & 2 \\
2011 & 2 \\
2010 & 6 \\
2009 & 2 \\
2008 & 1 \\
2007 & 2 \\
2006 & 1 \\
2005 & 2 \\
2004 & 1 \\
2003 & 1 \\
\hline
\end{tabular}

Fonte: Adaptada SCOPUS, 2020.

De acordo com os dados abordados na Tabela 14, o ano de 2019 foi o que contemplou o maior número de artigos publicados que continham em seus estudos "Brand e Education Insitution".

Além dos anos com maior número de publicação, também foram analisados os autores e o número de publicações. Ao analisar, observou-se que os autores obtiveram o mesmo número de publicações $(n=1)$.

Outra análise realizada consistiu no número de citações que os autores dispõem. 


\section{Figura 9 - Mapa de análise de citação}

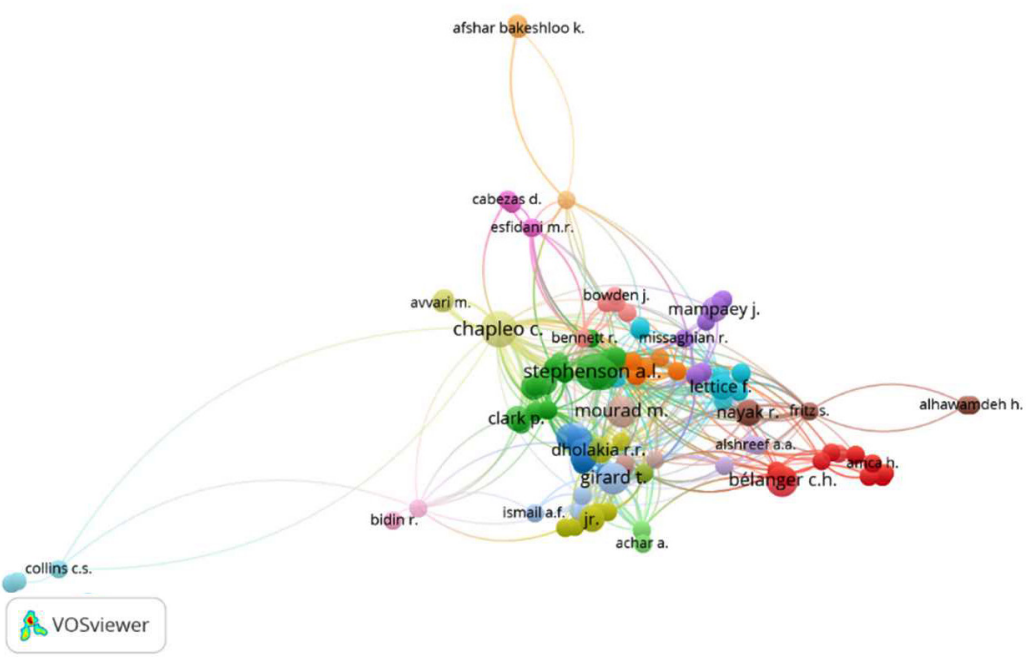

Fonte: Adaptada SCOPUS, 2020.

A Figura 9 mostra o mapa de citações dos autores que pesquisam Brand e Instituição de Ensino. Os autores que apresentam maior densidade no mapa, como o Lettice, F., são aqueles mais citados.

Tabela 15 - Autores mais citados

\begin{tabular}{l|cr}
\hline Autor & Publicações & Citações \\
\hline Agahi, H. & 1 & 183 \\
Matzdorf, F. & 1 & 183 \\
Price, I. & 1 & 183 \\
Smith, L. & 1 & 183 \\
Chapleo, C. & 4 & 154 \\
Lettice, F. & 2 & 99 \\
Rutter, R. & 2 & 99 \\
Ali-Choudhury, & & \\
R. & 1 & 80 \\
Bennett, R. & 1 & 80 \\
Chauhan, K. & 1 & 70 \\
\hline
\end{tabular}

Fonte: Adaptada SCOPUS, 2020.

Conforme exposto na Tabela 15, Agahi, H.; Matzdorf, F.; Price, I.; Smith, L. são os autores mais citados entre as pesquisas sobre Brand em Instituição de Ensino, com 183 citações cada e apenas uma publicação sobre o tema. 
Figura 10 - Mapa de coautoria

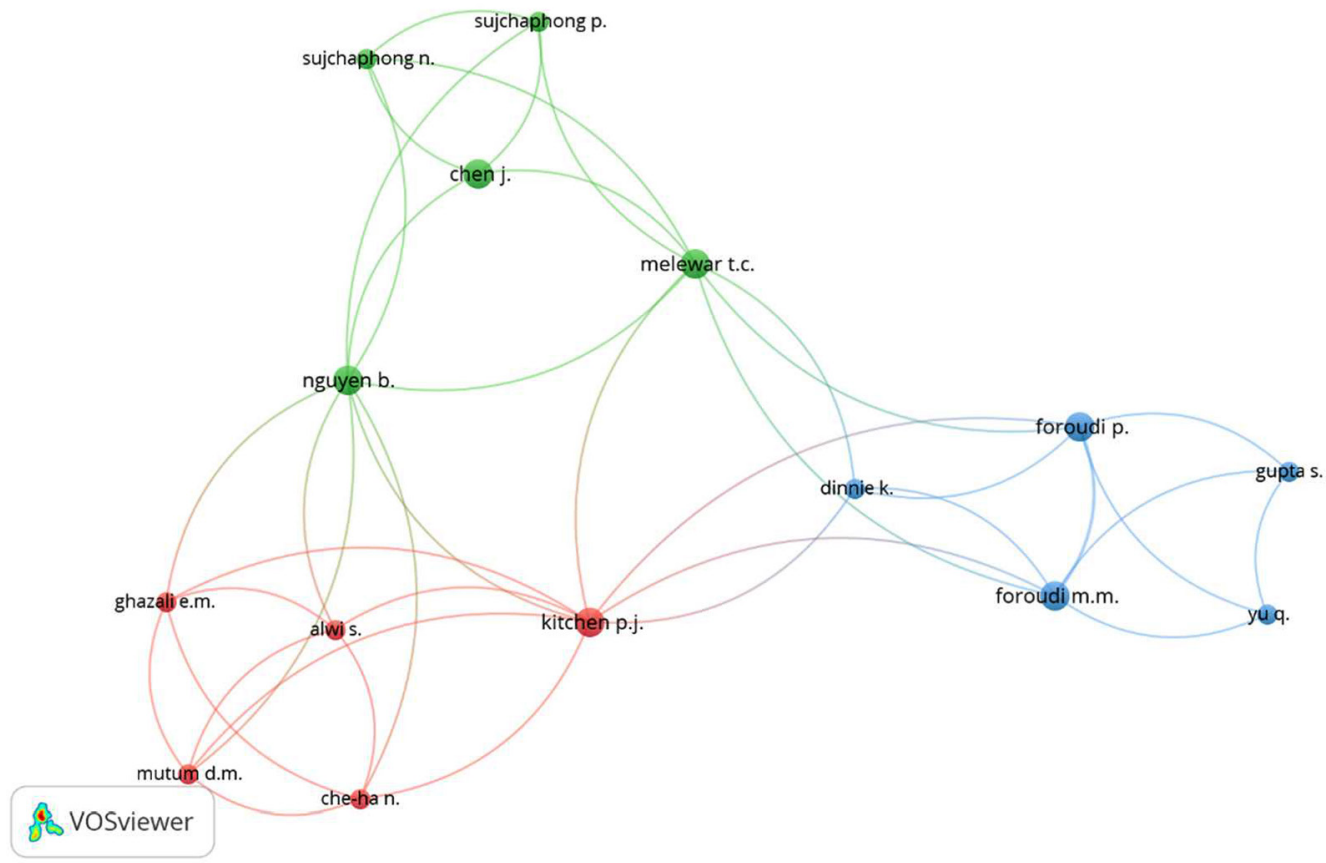

Fonte: Adaptada SCOPUS, 2020.

A partir da análise da Figura 10, observa-se que os autores realizam trabalhos em conjunto acerca da temática da marca em instituição de ensino.

Ao contemplar as palavras que co-ocorrem, a Figura 11 traz uma visão sistematizada.

\section{Figura 11 - Co-ocorrência de palavras}

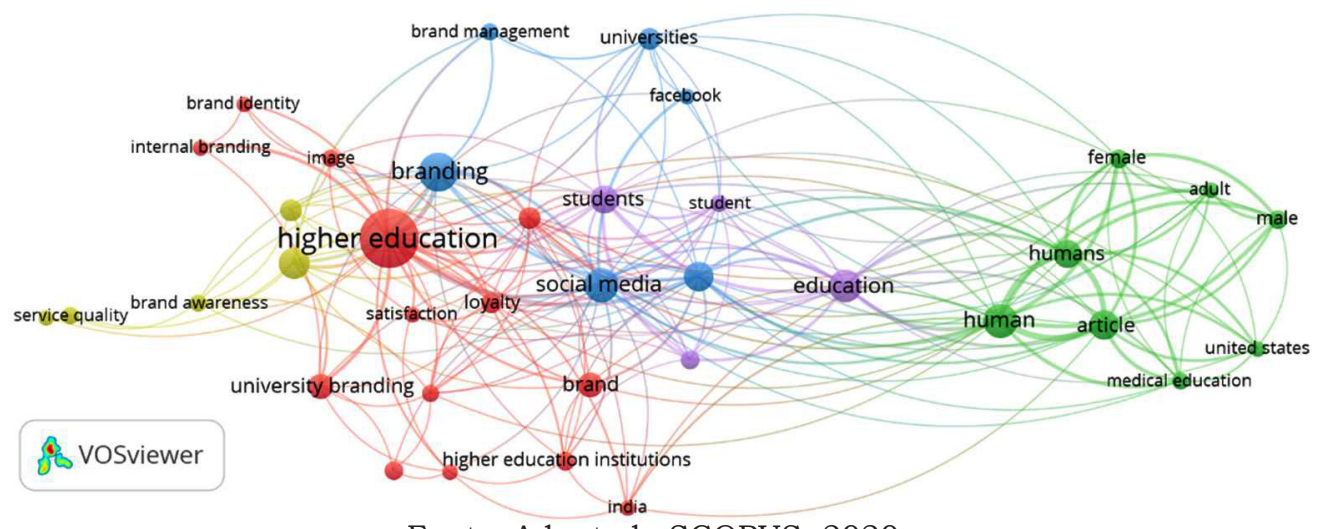

Fonte: Adaptada SCOPUS, 2020. 
Entre os artigos buscados, as palavras que mais co-ocorrem são: $H i$ gher education (66), branding (29), human (22) e social media (22). Diante do exposto, verifica-se que os estudos sobre a marca em Instituição de Ensino Superior contemplam os autores citados nessa análise bibliométrica.

\subsection{Palavra-chave Brand Management and Education Institution}

A busca pela palavra Brand Mangement and Education Insitution resultou em 131 documentos publicados entre 1984 e 2020, sendo 83 artigos publicados entre 2003 e 2020.

Tabela 16 - Número de publicações por ano

\begin{tabular}{c|r}
\hline Ano & $\mathbf{N}^{\mathbf{0}}$ de artigos publicados \\
\hline 2020 & 11 \\
2019 & 14 \\
2018 & 9 \\
2017 & 10 \\
2016 & 8 \\
2015 & 4 \\
2014 & 6 \\
2013 & 3 \\
2012 & 1 \\
2011 & 4 \\
2010 & 3 \\
2009 & 2 \\
2008 & 0 \\
2007 & 2 \\
2006 & 2 \\
2005 & 1 \\
2004 & 1 \\
2003 & 2 \\
\hline
\end{tabular}

Fonte: Adaptada de SCOPUS, 2020.

A Tabela 16 mostra o número de artigos publicados por ano, os quais contemplam a gestão da marca em instituição de ensino. Observa-se que os anos com maior número de publicações foram 2019 e 2020. Além disso, observa-se que este tema começou a ser discutido recentemente, em 2003. 
Figura 12- Análise de coautoria

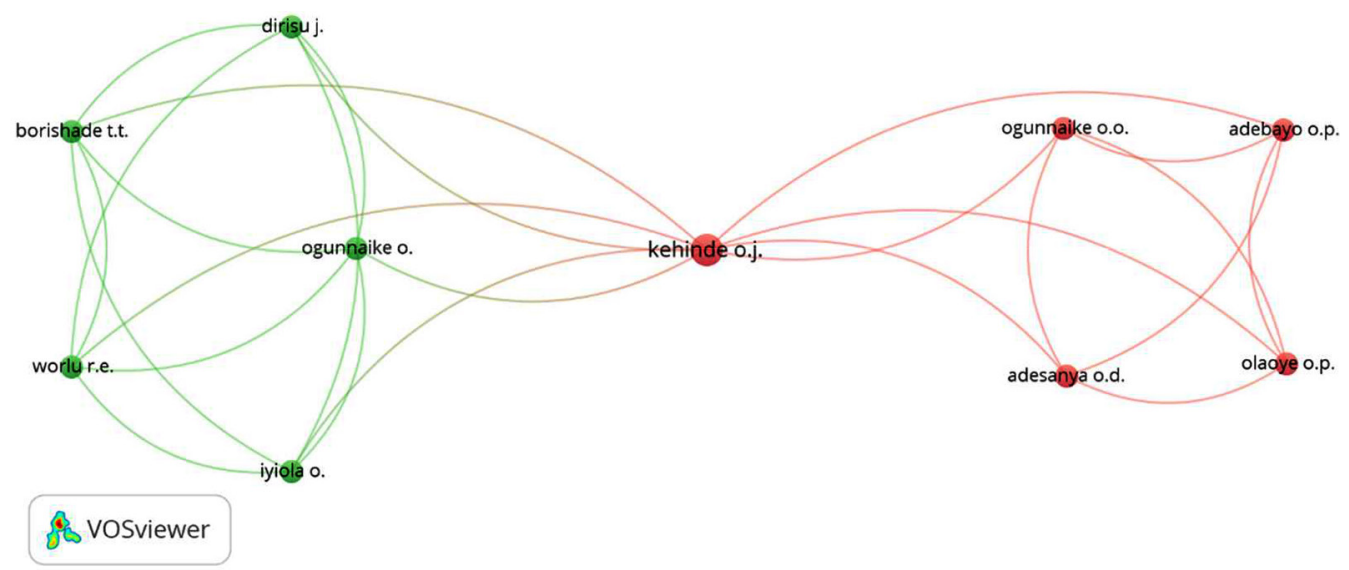

Fonte: Adaptada de SCOPUS, 2020.

A Figura 17 mostra os autores que trabalham em conjunto e, a partir da análise do mapa, observa-se que, entre os autores que pesquisam sobre o tema, apenas dez (10) trabalham em conjunto, tendo o Kehind, O. J. como o autor central das publicações.

Tabela 17 - Número de publicações por país

\begin{tabular}{l|r}
\hline Pais & Número de publicações \\
\hline United Kingdom & 13 \\
United States & 13 \\
India & 11 \\
Malaysia6 & 6 \\
Canada5 & 5 \\
Russian & \\
Federation & 4 \\
Australia & 3 \\
Nigeria & 3 \\
Spain & 3 \\
China & 2 \\
\hline
\end{tabular}

Fonte: Adaptada de SCOPUS, 2020.

A Tabela 17 mostra o número de publicações com a temática da gestão de marca em instituições de ensino em cada país no qual as pesquisas foram realizadas, com destaque ao Reino Unido $(n=13)$ e aos Estados Uni$\operatorname{dos}(\mathrm{n}=13)$. 
Figura 13 - Mapa de co-ocorrência de palavras

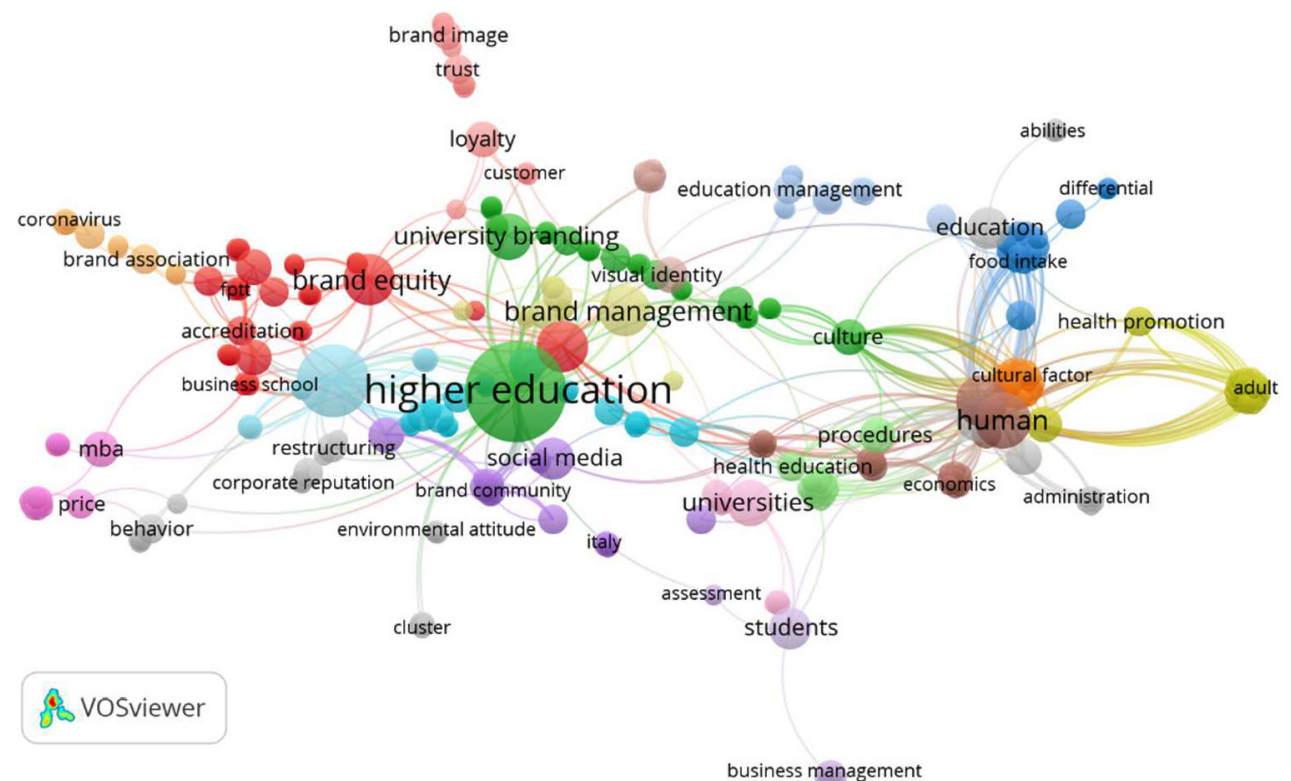

Fonte: Adaptada de SCOPUS, 2020.

A Figura 13 mostra a co-ocorrência de palavras nos artigos buscados, sendo Higer education; Human; University brand; Brand equity e outras as mais citadas.

Ao realizar a busca das palavras Brand hatred; Hate the Brand; e Education Institution não foram encontrados trabalhos publicados na base de dados Scopus Elsevier. Essa busca demonstra a necessidade de se realizar uma pesquisa voltada para a gestão da marca em Instituições de Ensino Superior (IES) sob a ótica do ódio à marca.

\section{Considerações Finais}

Entre os autores que mais publicaram com a temática, o King, C. ganha destaque desenvolvendo 15 estudos sobre gestão da marca como primeiro autor e também coautor, entre os anos de 2006 e 2020, sendo o seu estudo mais citado "O papel do envolvimento do cliente na construção da lealdade do consumidor para as marcas de turismo", o qual publicou em 2016, com autoria de So, K.K.F., King, C., Sparks, B.A., 
Wang, Y., no Journal of Travel Research. Este artigo contribuiu para 207 outras pesquisas sobre a temática, o que demonstra a sua importância para a academia.

Conforme abordado anteriormente, os estudos sobre a gestão da marca se mostram importantes devido ao número de publicações e também de contribuições para outras pesquisas, com as citações.

Ao contemplar a gestão da marca (brand management) em instituições de ensino, observa-se que as pesquisas sobre o tema foram iniciadas em 2009 e, até o momento, 20 trabalhos foram publicados, conforme bibliometria realizada a partir da base de dados Scopus.

Entre os autores que realizaram trabalhos sobre gestão da marca em instituição de ensino, Mourad, M. se destaca por obter o maior número de estudos sobre o tema, sendo os seus estudos voltados para a marca como influência de escolha do aluno (Mourad, 2011) e a internacionalização como estratégia de posicionamento de mercado de instituições de ensino superior (Mourad, 2010).

Mediante busca na base de dados Scopus, obteve-se 229 estudos sobre Branding em instituições de ensino, sendo o ano de 2020 destaque em número de publicações com a temática, contemplando 35 estudos.

Entre os trabalhos mais citados, o livro de Brown \& Carasso (2013) se destaca, tendo como título "Tudo à venda?: A mercantilização do ensino superior no Reino Unido", o livro contribuiu para mais 246 pesquisas. O estudo de Brown \& Carasso (2013) abordaram um período de aumento no número de matrículas no ensino superior e um momento de aumento da competitividade entre as IES, o que acarretou no que os autores chamam de mercantilização do ensino superior.

Assim como o estudo de Brown \& Carasso (2013), outros trabalhos abordam a gestão da marca de IES mediante um aumento de demanda por ensino superior e a necessidade de posicionamento de marca, sendo este comportamento das IES alvo de crítica, como feito por Weisbrod, Ballou e Asch (2008), que abordam artigos que criticam o fato das IES estarem cada vez mais voltadas para fins lucrativos e em conflito com a sua missão. 
Os dados coletados permitem inferir acerca da importância do estudo sobre o Branding, Gestão de Marca em Instituição de Ensino e as palavras-chave correlacionadas a essa temática: "Brand"; "Brand Management"; "Brand Hatred"; "Brand Loyalty"; "Brand and Educational Institution"; "Brand Management and Educational Institution".

Percebe-se que as temáticas aqui abordadas contribuem para a pesquisa acadêmica em quantidade, mediante número de publicações e relevância, mediante os trabalhos citados.

Não obstante, a análise bibliométrica também permitiu concluir que há uma lacuna ao observar a busca pelas palavras-chave Brand hatred; Hate the Brand; e Education Institution, as quais não constaram publicações na base de dados Scopus.

Diante dessa lacuna, sugere-se que sejam realizados estudos voltados para o ódio à marca e o ódio à marca em instituições de ensino e gestão da marca como fator de estratégia de retenção e promoção da lealdade e fidelização de alunos.

\section{Referências}

BROWN, R.; CARASSO, H. Everything for sale?: The marketisation of UK higher education. 1 Ed. Liverpool Hope University, United Kingdom. Department of Education, University of Oxford, United Kingdom. 2013. ISBN: 978-020307116-8.

MOURAD, M. Internationalisation: a new positioning strategy in the higher education market. International Journal of Management in Education 4(2), pp. 185-200, 2010.

MOURAD, M. Role of brand related factors in influencing students' choice in Higher Education (HE) market. International Journal of Management in Education 5(2-3), pp. 258-270, 2011.

SCOPUS. Base de Dados Scopus. Site Institucional. Disponivel em: https:// www-scopus.ez27.periodicos.capes.gov.br/search/form.uri?display=basic\#basic. Acesso em: $05 \mathrm{dez} 2020$. 
SO, K.K.F.; KING, C.; SPARKS, B.A.; Wang, Y. O papel do envolvimento do cliente na construção da lealdade do consumidor para as marcas de turismo. Journal of Travel Research, vol 55, Ed. 1, 2016.

WEISBROD, B.A.; BALLOU, J.P.; ASCH, E.D. Mission and Money: Understanding the University. $1 \mathrm{Ed}$. Institute for Policy Research, Northwestern University, Evanston, IL, United States. Mathematica Policy Research, Inc, Cambridge, MA, United States. 2008. ISBN: 978-051151101-1; 978052151510-8.

ZUFRYDEN, F. C. A Logit-Markovian Model Of Consumer Purchase Behavior Based On Explanatory Variables: Empirical Evaluation And Implications For Decision Making. Decision Sciences. University of Southern California, United States. Volume 12, Issue 4, Pages 645-660. Outubro 1981. 